\title{
Update to the \\ 1993 AMS Membership Directory
}

Listed below are corrections, changes and some additions to information published in the 1993 AMS Membership Directory. ${ }^{1}$ The American Meteorological Society would like to thank its members for providing these corrections and apologize for any inconvenience that these errors or omissions may have caused.

Due to an inordinate amount of errors in the geo- graphical section of the directory, a new list will be compiled and will appear in a future issue of the Bulletin of the American Meteorological Society.

In addition, we have been notified that the telephone exchange for members affiliated with the Naval Postgraduate School in Monterey, California has been changed from 646/647 to 656 .
Anderson, Lee C.

NWS

9607 NW Beaver Drive

Johnston, IA 50131

O: 515-270-4501

Arango, Hernan G.

Div. of Applied Sci.

Harvard Univ.

29 Oxford St., Cambridge, MA 02138

O: 617-495-3457 F: 617-495-5192

E: arango@ pacific.harvard.edu

Arya, Dr. S. Pal

Dept. Marine, Earth, Atmos. Sci.

NC State Univ.

Box 8208

Raleigh, NC 27695-8208

O: 919-515-7002 F: 919-515-8201

Assel, Raymond A.

2205 Commonwealth Blvd.

Ann Arbor, MI 48105-1593

O: 313-741-2268 F: 313-741-2055

E: ASSEL@GLERL.NOAA.GOV

Barnes, Dr. Arnold A., Jr.

Chief, Cloud Physics Section

Phillips Lab/GPAA

29 Randolph Road

Hanscom AFB, MA 01731-3010

O: 617-377-2939 F: 617-377-2984

E: BARNES@PLH.AF.MIL

'The 1993 AMS Mermbership Directory was published with the September 1993 issue of the Bulletin of the American Meteorological Society.

(C)1993 American Meteorological Society
Bauman, William H., III

US Air Force, NC State Univ.

MEAS, Box 8208

Raleigh, NC 27695-8208

O: 919-515-7803 F: 919-515-7802

E: bauman@measun.nrrc.ncsu.edu

Bergman, Kenneth $\mathrm{H}$.

NASA Headquarters, Code YSM

Office of Mission to Planet Earth

Washington, DC 20546

O: 202-358-0765 F: 202-358-2770

E: KBERGMAN@NASAMAIL.NASA.GOV

Bernier, Sally

WVAB

8443 Day Drive

Cleveland, $\mathrm{OH} 44112$

Bluestein, Howard B.

School of Meteor.

Univ. of Oklahoma

100 E. Boyd Rm 1310

Norman, OK 73019

E:hblue@metgem.gcn.uoknor.edu

Brittig, Jeffrey S.

USAF

Randolph AFB, TX 78148

O: $210-652-4826$

Brown, James S., Jr.

210 Edward Street

Kingsport, TN 37665-1609

Browning, Dr. Keith A.

Dir. Jt. Ctr. Mesoscale Meteorology

Harry Pitt Bldg.

Univ. of Reading

PO Box 240

Reading, Berkshire, UK RG6 2FN

O: $44734316521 \mathrm{~F}: 44734318791$
Burnett, Paul T.

1826 Lamar Circle

Alamogordo, NM 88310

Busby, Bryan T., TV Seal \#425

KMBC-TV, 1049 Central

Kansas City, MO 64105

Cantin, Jean-Guy

100 Boul. Alexis Nihon, Ste. 300

St. Laurent, Quebec, Canada H4M 2N8

Chen, Jen-Ping

C4/SIO/0239

La Jolla, CA 92093-0239

O:619-534-8290 F: 619-534-4922

E: jpchen@fiji.ucsd.edu

Cheng, Dr. Chi-Shung

Hughes STX

Room 365

7701 Greenbelt Road

Greenbelt, MD 20770

O: 301-441-4103 F: 301-441-2392

Cleaveland, Malcolm K.

Dept. of Geography

Ozark Hall 108A

Univ. of Arkansas

Fayetteville, AR 72701

Collier, Dr. C.G.

Metstar Consult., Meteorological Office

Sutton Hse. Rm G6, London Rd.

Bracknell, Berkshire RG1225Y

United Kingdom

O: +44344856244 F: +44344854024

E:ccollier@email.meto.govt.uk

Cooley, Duane S.

4503 Libbey Drive

Fairfax, VA 22032 
Croft, Dr. Paul J.

Univ. of South Alabama

Dept. of Geology \& Geography

LSCB 341

Mobile, AL 36688-0002

O: 205-460-6381 F: 205-460-7886

E: usapjc@asnusa.asn.net

Deblonde, Dr. Godelieve

Aerospace Meteor. Div.

Atmos. Envir. Serv.

2121 Transcanadian Hwy., 5th $\mathrm{fl}$.

Dorval, Quebec, Canada H9P 1J3

O: 514-421-4774 F: 514-421-2106

E: GDEBLONDE@RPN.AES.DOE.CA

Derome, Dr. Jacques

Dept. Atmos.\& Oceanic Sci.

McGill Univ.

805 Sherbrooke St. W

Montreal, Quebec, Canada H3A 2K6

O: 514-398-3766 F: 514-398-6115

E:DEROME@ZEPHYR.METEO.MCGILL.CA

DiVecchio, Tom

WJET-TV, 8455 Peach Street

Erie, PA 16509

O: $814-864-2400$

Drag, Walt

445 Myles Standish Blvd.

Taunton, MA 02780

O: 508-823-2087 F: 508-823-2321

Duce, Dr. Robert, Dean

Coll. Geosci. \& Maritime Studies

Texas A\&M Univ.

College Station, TX 77843

E: R.DUCE [OMNET]

Elliot, Cynthia A.

Amoco Corp.

200 E. Randolph Dr.

Mail Code \#4901

Chicago, IL 60601

O: 312-856-6193 F:312-616-0985

Fago, Karen S.

NWS

2500 Challenger Drive

Midland, TX 79706

O: 915-563-3713

Farrell, Brian F.

Dept. of Earth \& Planetary Sci.

Harvard Univ.

Pierce Hall

Cambridge, MA 02139

E: bff@io.harvard.edu

Fields, Robert M.

Commanding Officer

Mar. Corps Det.

824 Hercules St., Ste. 104

Keesler AFB, MS 39534-2099

O: 601-377-7385 F: 601-337-7393
Fischer, Robert E.

NWS/FO

1 12th Ave., Box 21

Fairbanks, AK 99701

O: 907-456-0247 F: 907-456-0381

Fishbein, Emanuel B.

140-72 Burden Crescent

Briarwood, NY 11435

Fishel, Gregory B.

P.O. Box 12000

Raleigh, NC 27605

O: 919-821-8590, F: 919-821-8541

Fisher, George E.

Engineering Science

3175 Rte. 94

Woodbine, MD 21797

Fisher, Maj. George F.

105 Littleton Road

Chelmsford, MA 01824

Fisher, Lonnie W.

7255 Seven Mile Road

Freeland, MI 48623

Fisher, Luther F.

11713 Shoshone Drive

Austin, TX 78759

Fisher, Perry W.

Dames \& Moore

One Cont. Towers

17 Golf Road, \#1000

Rolling Meadows, II 60008

O: 708-228-0707 F: 708-228-1115

Fisher, Richard W.

Rocky Mtn. Forest \& Range Exp. Sta.

240 West Propect Rd.

Ft Collins, CO 80526

O: 303-498-1232 F: 303-498-1010

Fisher, Robert G.

6941 N. Camino de Fosforo

Tucson, AZ 85718-1011

O: 602-797-2877 F: 602-797-2877

Fisher, William J., Jr.

2049 Schubert Drive

Virginia Beach, VA 23454

Fishman, Aaron J.

13149 Pavilion Lane

Fairfax, VA 22033

O: 703-378-7227

E: Compuserve $(72607,613)$

Fishman, Dr. Jack

NASA/Langley Res. Center

Mail Stop 4a

Hampton, VA 23665-5225

O: 804-864-2720, F: 804-864-7790

E: fishman@asdsun.Larc.Nasa.Gov
Fisk, Charles J.

Pacific Missile Test Ctr.

Point Mugu Nas, CA 93042

O: 805-989-8383

Fitch, Dr. Bruce Wesley

3319 Quimby St.

San Diego, CA 92106

O: 619-592-5357

Fitch, Scott R.

1039 Whalen Road

Penfield, NY 14526

Fitzgerald, Ann E.

5 Nabby Road, Unit A-56

Danbury, CT 06811

Fitzgerald, David B.

914 S. 2nd St.

Laramie, WY 82070

Fitzgerald, Dr. Donald R.

71 Jennie Dugan Road

Concord, MA 01742

Fitzgerald, Dr. James W.

Naval Research Lab. Code 7228

Washington, DC 20375

E:FITGERA@CCFYMPEL.NRL.NAVY.MIL

Fitzgerald, Jay S.

USAF

698 Korina St.

Vandenberg AFB, CA 93437

Fitzner, Craig A.

Michigan Dept. of Natural Resources

Air Quality Div., P.O. Box 30028

Lansing, MI 48909

O: 517-373-7044 F: 517-373-1265

Fitzpatrick, Michael E.

109 Gregg Place Circle

Bellevue, NE 68005-4956

Fiuza, Prof. Armando F. G.

Lisbon Univ.

Physics Dept.

Rua Escola Politecnia 58

1200 Lisbon, Portugal

Flagg, Charles $\mathrm{N}$.

Brookhaven National Lab.

Bldg. 318

Upton, NY 11973

O: 516-282-3128 F: 516-282-2060

E: C.Flagg [OMNET]

Flaherty, James E.

6 Park Drive

Woburn, MA 01801

Flaherty, Paul E.

19C Apple Lane

Hollis, NH 03049 
Flahive, A. C.

4 Fairfax Ave.

Apt. \#284

Derry, NH 03038

Flament, Pierre J. 1000 Pope Road

Honolulu, HI 96822

Flanders, Allen F. 15208 Candytuft Lane Rockville, MD 20853

O: 301-871-6566

Flannigan, Michael D.

P.N.F.I.

Chalk River, Ontario

Canada KOJ 1JO

Flatley, Jon M.

1730 Towpath Road

Dauphin, PA 17018

O: 301-763-8493

Flattery, Col. Thomas W.

3 Hacienda

Irvine, CA 92720

O: 310-942-3934

Fleagle, Dr. Robert G.

Dept. of Atmos. Sci.

Univ. of Washington

Seattle, WA 98195

O: 206-543-4594 F: 206-543-0308

Fleisher, Dr. Aaron

M. I. T.

Room 9-549

Cambridge, MA 02139

O: 617-253-7497 E:

afleishr@ atheur.Mitedu

Fleming, Eric L.

Applied Research Corp.

82 Corporate Drive

Landover, MD 20785

O: 301-286-3923 F: 301-286-3460

E: fleming@sgccp (internet)

Fleming, Jack N. Jr.

Piedmont, Olsen, Hensley

216 West 8th St., Ste. 400

Chattanooga, TN 37402

O: 615-756-7193 F: 615-756-7197

Fleming, James R.

Dept. of Physics \& Astronomy

Colby College

Waterville, ME 04901

O: 207-872-3548

Fleming, Julius D.

350 Palisade Drive

Florence, AL 35630-5936
Fleming, Dr. Rex J.

NCAR

P.O. Box 3000

Boulder, $\mathrm{CO} 80307$

O: 303-497-8165 F: 303-497-8181

E: fleming@mmm.Ucar.Edu

Flenz, Edward R.

NWS/NOAA

Kent County Airport

Grand Rapids, MI 49512

O: 616-456-2268

Flesch, Thomas K.

Dept. of Geography

Univ. of Alberta

Edmonton, Alberta

Canada T6G 2H4

Fletcher, John E.

58 E. Village Loop Road

Ponoma, CA 91766-4839

Gaffen, Dr. Dian J.

NOAA Air Res. Lab

1315 E. W. Hwy., Rm. 3151

Silver Spring, MD 20910

Gommel, Brig. Gen. William R.

23 Lawrence Avenue

Indianapolis, IN 46227-8635

Grassotti, Christopher

Atmos. Environ. Research Inc.

840 Memorial Dr.

Cambridge, MA 02139

O: 617-547-6207 F: 617-661-6479

E: CGRASS@AER.COM

Hadley, David S.

2066 E. Lonsdale Drive

Salt Lake City, UT 84121

Halblaub, Jeffrey D.

Ohio State University

2072 Baker Hall, 129 W 12th Ave.

Columbus, $\mathrm{OH} 43210$

O: 614-293-1145 E: halblaub.1@osu.edu

Harman, Donald $P$.

KIMT-TV3

112 N. Pennsylvania

Mason City, IA 50401

O: 515-423-2540 F: 515-423-9309

Hauth, Floyd F.

TASC, 11 Wilson Blvd., Suite 1500

Arlington, VA 22209

O: 703-358-9090 X6464 F: 703-524-6666

E: ffmhauth@tasc.com

Heim, Richard R., Jr.

NOAA/NCDC, Federal Bldg., MS42

Ashville, NC 28801

O: 704-271-4251

E: RHEIM@NCDC.NOAA.GOV
Holland, Joshua Z.

13211 Bluhill Road

Wheaton, MD 20906

Horne, Christopher S.

NWS

250025 Airport Terminal St.

Scotts Bluff, NE 69361-9517

O: 308-632-6822

Hsieh, Dr. William

Dept. of Oceanography

Univ. of British Columbia

Vancouver, B.C. Canada V6T 1 Z4

O: 604-822-2821 F: 604-822-6091

E: william@ocgy.ubc.ca

Kavinsky, Marc B.

NWS/NOAA

N3533 Hardscrabble Rd.

Dousman, WI 53118

O: 414-965-2765 F: 414-965-4296

Kleespies, Thomas J.

WWB Room 810

NOAA/NESDIS E/RA14

Washintong, DC 20233

O: 301-763-8136 F: 301-763-8108

E: Kleespies@NZMS.WWB.NOAA.GOV

Koch, Nicolle E.

8405 Navigation Drive

Rowlett, TX 75088

Koffler, Russell

12200 Hollybank Drive

Fort Washington, MD 20744

E: RKOFFLER@AOL.COM

Kvamsto, Dr. Nils G.

Geophysical Ins., Univ. of Bergen

Allegaten 70, N5007, Bergen, Norway

O: +4755212638 F: +4755960566

E: nilsg@gfi.uib.no

Lacy, Stanley J.

9 Monticello Drive

Lake Oswego, OR 97035

Lally, Susan K.

Seimac Res. Ltd./271 Brownlow Ave.

Dartmouth, Nova Scotia,

Canada B3B 1W6

O: 902-468-3007 F: 902-468-3009

Lare, Dr. Andrew R.

NASA/GSFC/Applied Research Corp.

Code 913

Greenbelt, MD 20771

O: 301-286-9588 F: 301-286-1759

Lee, Martin E.

US Army Resear. Lab.

Battlefield Environment Directorate

WSMR, NM 88002

F: 505-678-0343 E: mlee@ arl.army.mil 
Mazuk, Ann L.

The Aerospace Corp.

PO Box 92957, M2/266

Los Angeles, CA 90009-2957

O: 310-336-5000 F: 310-336-1636

E: ANN_MAZUK@QMAIL2.AERO.ORG

Mazzola, Carl A.

Stone \& Webster South Operation 1080 Silver Bluff Rd., Ste. E

Aiken, SC 29803

O: 803-643-7619 F: 803-643-7610

Meier, Keith W.

NWS/Wstrn. Reg. Hdqtrs.

Sci. Serv. Div. W/WR3x4

P.O. Box 11188

Salt Lake City, UT 84147

O: 801-524-5131 F: 801-524-5246

E: KM1036\%albnyATM.bitnet@UACSC2.

ALBANY. edu

Meyer, Michael B.

Rupprecht \& Patashnick Co., Inc.

25 Corporate Circle

Albany, NY 12203

Nelson, Dale A.

PSC \#1, Box 2602,

Fairchild AFB, WA 99011

O: 509-244-4565

Nitz, Bradley W.

WCTI-TV, P.O. Box 12325

New Bern, NC 28561

O: 919-636-1212 F: 919-636-6855

Noah, Daniel G.

NOAA, Weather Serv. Forecast Office

P.O. Box 1016,

Bismarck, ND 58502-1016

O: 701-250-4224 F: 701-250-4450

E: CompuServe ID\# 70673,144

Olivier, Michael G.

Comp. Sci. Raytheon

Meteo. CSR 4140, P.O. Box 4127

Patrick AFB, FL 32925-0127

O: 407-853-8289 F: 407-853-8291

Orr, Brad W.

NOAA/ERLWPL, RE/WP6

325 Broadway

Boulder, CO 80303

O: 303-497-5152 F: 303-497-6978

E: bwo@wpl.erl.gov

Pearson, Allen

260 Terrace Trail W

Lake Quivira, KS 66106

Pell, Dr. Jerry

Sr. Envir. Sci./Fossil Energy

FE222/US Dept of Energy (270 CC)

Washington, DC 20585

O: 301-903-9447 F: 301-903-9438
Qiu, Bo

Univ. of Hawaii at Manoa

Dept. of Oceanogr.

1000 Pope Rd.

Honolulu, HI 96822

O: 808-956-9505 F: 808-956-9225

E: bo@ iniki.soest.hawaii.edu

Richmond, Ken

McCulley, Frick \& Gilman Inc.

3400 188th St SW, Ste. 400

Lynnwood, WA 98037-4708

O: 206-778-8252 F: 206-771-8842

Rolph, Glenn D.

NOAA/ARL, 1315 East West Hwy.

Silver Spring, MD 20912

O: 301-713-0295 F: 301-713-0119

E:GLENN@ARLRISC.SSMC.NOAA.GOV

Salkovitz, Daniel D.

Virginia Dept. of Envir. Quality

Air Div.

P.O. Box 10089

Richmond, VA 23240-0089

O: 804-786-6851 F:804-225-4464

Sanger, Gary
NWS
1400 Boughton Drive
Bakersfield, CA 93308
O: $805-861-4323$

Schwerdt, Richard W.

NWS/NOAA/Cntrl. Regn. Hdqtrs

6 E. 12th St

Kansas City, MO 64106-2897

O: 816-426-3239 F: 816-426-3270

Schwiesow, Dr. Ronald L.

NCAR/ATD/RSF/PO Box 3000

Boulder, CO 80307

O: 303-497-2061 F: 303-497-2044

E: schwies@ncar.ucar.edu

Shih, Chi Fan G.

NCAR

Scientific Computing Division

P.O. Box 3000

Boulder, CO 80307

O: 303-497-1833 F: 303-497-1137

E: chifan@ncar.ucar.edu

Smith, Kevin E.

U.S. Dept. of Commerce

NOAA/NWS

P.O. Box 5779

Jackson, MS 39288-5779

O: 601-936-2189

Smith, Col. William C.

Code ME/HQ NASA

300 E St. SW

Washington, DC 20546

O: 202-358-4466 F: 202-358-2820

E: bsmith@osfmsi.hq.nasa.gov
Spratt, Scott M.

NOAA/NWSO Melbourne

421 Croton Road

Melbourne, FL 32935

O: 407-254-6083

Srivastava, Bhartendu

AES Ontario Reg.,

Issues \& Development Branch

25 St. Clair Avenue, 8th Floor

Toronto, Ontario,

Canada M1V $3 A 3$

O: 416-973-5820 F: 416-973-1161

Stevens, Herbert

The Skiing Weatherman

39 Surrey Lane

N. Kingstown, RI 02852

O: 401-884-3206

Sullivan, Thomas J.

ARAC Prog. Leader

LLNL Box 808

Livermore, CA 94550-0808

O: 510-422-1838 F: 510-423-4908

E: TSULLIVAN@LLNL.GOV

Tarver, Edmund C., Jr.

2952 Colony Court,

Gainsville, GA 30507

Timchalk, Andrew

6503 Summerhill Road

Temple Hills, MD 20748

O: 301-449-4060

Tobin, William $\mathrm{H}$.

Fed. Aviation Adm.

Juneau AFSS

9230 Cessna Dr.,

Juneau, AK 99801

O: 907-463-4205

Trost, James E.

Oregon Dept. of Forestry

2600 State Street

Salem, OR 97310

Tucker, Dr. Donna F.

Dept. of Phys. \& Astron.

Univ. of Kansas

Lawrence, KS 66045

O: 913-864-4738 F: 913-864-5262

E: tucker@walrus.phsx.ukans.edu

Vanderbijl, Dr. Willem

Dept. of Meteorology

NPS

Monterey, CA 93943-5000

Walker, Wayland J.

Denver Planning Office, Rm 203

200 W. 14th Avenue

Denver, CO 80204

O: 303-640-4783 F: 303-572-4636 
Wang, Yinong

Atmospheric Sci. Programme

Dept. of Geography

Univ. of British Columbia

217-1984 West Mall

Vancouver, B.C., Canada V6T 1W5

O: 604-822-2269 F: 604-802-6150

Waunsch, John Gerard

WPLG-TV (Post-Newsweek)

3900 Biscayne Blvd.

Miami, FL 33137

O: 305-325-2469 F: 305-325-2480

White, Dr. G. A. III

P.O. Box 45826

Oklahoma City, OK 73145

O: 405-739-7617 F: 405-739-7403

Wolff, Paul M.

1150 Mestres Dr.

Pebble Beach, CA 93953-2928

Wood, Jeffrey L.

OR Army Nat. Guard, Dept. 1, 2nd Bn

218th Field Artil., 12th \& Belmont

Hood River, OR 97031

O: 503-386-3161

Woodman, Michael

EA Engin., Sci. \& Tech. Inc.

11019 McCormick Rd.

Hunt Valley, MD 21031

O: 410-584-7000 F: 410-771-1625
Zylkuski, Gary M.

Fleetweather Ocean Serv. Inc.

1966 Rte. 52

Hopewell Jct., NY 12533

O: $914-226-8400$ F: $914-226-4210$

Berman, Stephen

O: 607-436-3069

Bingham, Frederick $M$.

E: F.BINGHAM@SOEST.HAWAII.EDU

Boyer, Douglas

O: 304-256-2833 F: 304-256-2921

Burch, Larry E.

O: 801-524-5692 F: 801-524-6767

Fathauer, Theodore F.

F: 907-456-0381

Gilhousen, David B.

O: 601-688-2840 F: 601-688-3153

E: DAVEG@NDBC.SSC.NASA.GOV

Hoff, Raymond M.

O: 705-458-3310 F: 705-458-3301

E: RHOFF@CID.DOE.AES.CAJ

Kandel, Robert S.

O: $33169334543 \mathrm{~F}: 33169333049$

E:KANDEL@LMDX04.POLYTECHNIQUE.

FR
Kessler, Edwin

E:EKESSLER@METGEM.GCN.UOKNOR.

EDU

LeBlanc, Lynn L.

F: 318-342-1857

E: GELEBLANC@MERLIN.NLU.EDU

Mefford, Thomas $\mathrm{K}$

F: 303-497-6290

E: tmefford@cmdL2.cmdL.erL.GOV

Peterson, Dr. James T.

E: jim@ccg.cmdl.erl.gov

Toth, Zoltan

O: 301-763-8161 F: 301-763-8545

E: TOTH@SUN1.WWB.NOAA.GOV

Washington, Warren M.

E:WMW@NCAR.UCAR.edu

The Contact person for AccuWeather by correction will be:

Steinberg, Michael A.

AccuWeather Inc.

619 W. College Avenue

State College, PA 168601

\section{EMPLOYMENT ANNOUNCEMENTS}

\section{The American Meteorological Society publishes a monthly newsletter announcing employment opportunities for positions in the atmospheric and oceanic fields.}

Members and nonmembers may subscribe to the announcements at an annual (12 monthly issues) subscription rate of $\$ 15$ for AMS members and $\$ 30$ for nonmembers (Both members and nonmembers, kindly add $\$ 10$ for airmailing to a foreign address). After July 1, half-year subscriptions (for July through December issues) are available at one half of the above annual prices

Organizations with openings to be filled by qualified individuals seeking positions in the atmospheric and related fields should contact the American Meteorological Society, 45 Beacon Street, Boston, MA 02108-3693, 617-2272425 for announcement guidelines and charges. ASCII diskettes are invited. You may also submit announcements via internet: amsempl@aip.org. 